\title{
Relationship between Retroviral Replication and RNA Interference Machineries
}

\author{
K. Moelling, A. Matskevich, AND J.-S. JunG \\ Institute of Medical Virology, University of Zurich, CH-8006 Zurich, Switzerland
}

\begin{abstract}
Small interfering RNAs (siRNAs) associated with gene silencing are cellular defense mechanisms against invading viruses. The viruses fight back by suppressors or escape mechanisms. The retroviruses developed a unique escape mechanism by disguising as DNA proviruses. An evolutionary relationship between the siRNA machinery and the replication machinery of retroviruses is likely. The RNA cleavage enzymes PIWI and RNase H proteins are structurally related. This relationship can be extended from structure to function, since the retroviral reverse transcriptase (RT)/RNase $\mathrm{H}$ can also cause silencing of viral RNA by siRNA. Thus, both enzymes can cleave RNA-DNA hybrids and double-stranded RNA (dsRNA) with various efficiencies shown previously and here, demonstrating that their specificities are not absolute. Other similarities may exist, for example between PAZ and the RT and between RNA-binding proteins and the viral nucleocapsid protein. Dicer has some similarities with the viral integrase, since both specifically generate dinucleotide 3 '-overhanging ends. We described previously the destruction of the human immunodeficiency virus (HIV) RNA by a DNA oligonucleotide ODN (oligodeoxynucleotide). Variants of the ODN indicated high length and sequence specificities, which is reminiscent of siRNA and designated here as "siDNA." Cleavage of the viral RNA in the presence of the ODN is caused by the retroviral RT/RNase H and cellular RNase H activities. Several siRNA-mediated antiviral defense mechanisms resemble the interferon system.
\end{abstract}

We have previously designed an ODN targeted to the polypurine tract (PPT) of HIV with the idea of inhibiting HIV replication. The ODN was designed as a clamp around the $\mathrm{PPT}$ to inhibit the $\mathrm{RT} / \mathrm{RNase} \mathrm{H}$ during retroviral replication. The sequence consisted of an antisense strand homologous to the extended 25-nucleotide PPT. The second strand was designed on the basis of Hoogsteen base-pairing to allow a triple helix at the PPT. A linker of four nucleotides connected the strands. The PPT is the site that resists RNase $\mathrm{H}$ digestion during reverse transcription of the first cDNA copy, then the RT initiates at this local hybrid the second cDNA strand including cleavage by the RNase $\mathrm{H}$ at the ACU site, where two non-purines interrupt the PPT (Jendis et al. 1996, 1998; see Coffin et al. 1997).

The ODN, which by itself can form a hairpin-loop structure, was applied to $\mathrm{T}$ lymphocytes 2 hours postinfection with HIV at a low multiplicity of infection of 0.01 with ODN at $1 \mu \mathrm{M}$ concentration without carrier. This resulted in inhibition of viral replication for 20 days without viral breakthrough (Jendis et al. 1996). Patient isolates including resistant isolates against azidothymidin (AZT) were also inhibited. A control antisense ODN was less efficient (Jendis et al. 1996, 1998). Replication of two viral isolates, HIV IIIB and BaL-1, which differ in their PPTs by 2 of 24 or 23 nucleotides, was inhibited preferentially by their homologous ODNs and to a lesser extent by their heterologous ODNs. This indicates a very high sequence specificity (Moelling et al. 2006). The PPT is highly conserved, and 1700 of 2000 isolates collected in the Los Alamos Database were identical. PPT is also conserved in SIV (simian immunodeficiency virus).

We analyzed numerous variants of the ODN by changing single nucleotides or its length. All variants were ineffective in destroying the viral RNA (Moelling et al. 2006). To understand the molecular mechanism, we performed a kinetic analysis. RT-PCR (polymerase chain reaction) analysis indicated the disappearance of the viral RNA within about 2 hours posttreatment and the absence of a detectable DNA provirus (Jendis et al. 1996; Moelling et al. 2006). Furthermore, we were unable to demonstrate a triple-helix effect. However, we could attribute the ODNmediated molecular mechanism to destruction of the viral RNA by the retroviral RT/RNase H. We showed this by applying the ODN to purified viral particles, where the RT/RNase $\mathrm{H}$ was able to destroy the viral RNA and abolish viral infectivity within a few hours (Matskevich et al. 2006). The phenomenon is reminiscent of siRNA-mediated silencing and may be designated as "siDNA."

\section{SUBSTRATE SPECIFICITIES OF RNASE H AND PIWI}

siRNA-mediated silencing has been shown by dsRNA, 23-mers with overhanging ends, which are generated by Dicer and processed by AGO2 and associated proteins. The cleaving PIWI domain of Argonaute was shown to be structurally related to an RNase H (Song et al. 2004; Ma et al. 2005; Yuan et al. 2005). This relationship was only deducible from structural analyses and not obvious from the primary sequences. RNases $\mathrm{H}$ share a characteristic conserved triad of acidic amino acids at their active centers, the DDE motif (Yang and Steitz 1995; Nowotny et al. 2005). Not only do RNases H exist in retroviral replication complexes (Moelling et al. 1971; Moelling 1975; Hansen et al. 1988), but they also exist in Escherichia coli and mammalian cells as RNase $\mathrm{H} 1$ for removal of RNA primers during DNA replication (Nowotny et al. 2005). All of these RNase $\mathrm{H}$ enzymes cleave RNA in RNA-DNA hybrids (Moelling et al. 1971) or at the junction of RNA-DNA, opposite to an RNA strand, for example, dur- 

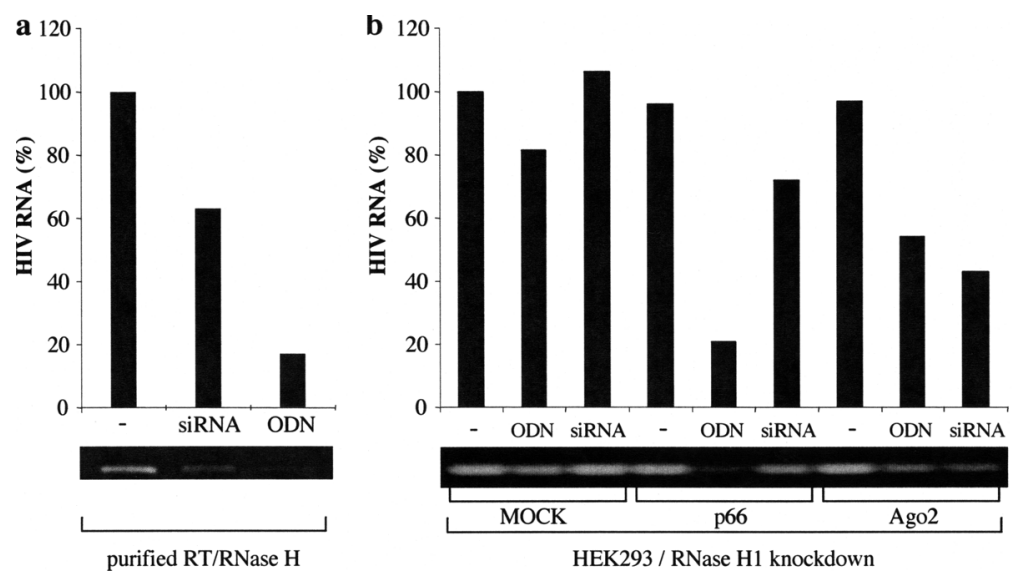

Figure 1. (a) Recombinant HIV RT/RNase H can cleave viral RNA in the presence of siRNA and an ODN, both targeted to the PPT of HIV RNA and measured by RT-PCR. (b) Cleavage of viral RNA in the presence of siRNA and ODN with cellular extracts as source of enzymes. Extracts were prepared from HEK 293 cells in which the cellular RNase H1 had been knocked down and either the p66 HIV RT/RNase $\mathrm{H}$ or the AGO2 protein was overexpressed. Cleavage was determined by RT-PCR.

ing HIV replication for removal of the tRNA primer after initiation of cDNA synthesis. The structural homology was, however, not shared at the functional level, because a hallmark of an RNase $\mathrm{H}$ is its specificity for cleaving RNA in RNA-DNA hybrids, not dsRNA as required for siRNA. Yet, both the RT/RNase $\mathrm{H}$ and PIWI give rise to oligonucleotides with 3'-OH and 5'-phosphate.

The PIWI domain has been shown to also cleave RNA in RNA-DNA hybrids, tested with an archaebacterial PIWI under extreme conditions, whereby the relative $K_{\mathrm{D}}$ values of single-stranded DNA to DNA-RNA hybrid to dsRNA were approximately 0.01 to 1 to 10 (Yuan et al. 2005).

We used the recombinant HIV-RT/RNase $\mathrm{H}$ with the ODN and siRNA and targeted them to the PPT of HIV together with HIV RNA extracted from viral particles in an in vitro reaction and then used primer sets covering the HIV-PPT RNA for analysis of cleavage of the RNA by RT-PCR analysis (Fig. 1a). As shown, RT/RNase H can perform siRNA-mediated silencing of HIV RNA, whereby the effect induced by the ODN is more efficient.

We then analyzed cells in which we had knocked down the cellular RNase $\mathrm{H} 1$ to more specifically determine the effect caused by the viral RT/RNase H. The cells, in which the cellular RNase H1 was knocked down, were transfected and overexpressed the HIV RT/RNase $\mathrm{H}$ and AGO2 (both linked to HA tags). In vitro reactions were performed with HIV RNA and ODN or siRNA, supplemented with the cellular extracts. As shown, the recombinant p66 RT/RNase H cleaved the viral RNA in the presence of ODN, and AGO2 cleaved the viral RNA in the presence of siRNA. Both enzymes also cleaved the RNA under reversed conditions, indicating that the RT/RNase $\mathrm{H}$ can silence RNA with low efficiency also by means of siRNA and AGO2 also by ODN (Fig. 1b). The results indicate that the specificities of the RT/RNase $\mathrm{H}$ and $\mathrm{AGO} 2$ are not absolute.

It should be noted that the HIV RT/RNase $\mathrm{H}$ and the murine leukemia virus (MLV) RT/RNase $\mathrm{H}$ have also been shown to process dsRNAs, not only RNA-DNA hybrids (Ben-Artzi et al. 1992; Hostomsky et al. 1994; Blain and Goff 1993). This activity has also been named RNase H*. Furthermore, a mutation in the active center of an archaeal
RNase H, D125N, resulted in the loss of dsRNA cleavage efficiency, but not for hybrids (Ohtani et al. 2004). In addition, stalling the RNase $\mathrm{H}$ by omission of the four desoxyribonucleoside triphosphates (4dNTPs), so that the RT cannot move and polymerize, increased the efficiency for cleavage of dsRNA (Götte et al. 1995). The relationship between RT/RNase H and PIWI is therefore both structural and functional, not just structural. Figure 2 summarizes these results, showing both homologous and heterologous activities. Destruction of the HIV RNA by the RT/RNase H in the presence of the "siDNA" is designated as "suicide," since a normal step in retroviral replication is mimicked by the siDNA, but prematurely, before a cDNA is made.

\section{COMPARISON OF RETROVIRAL AND RNA INTERFERENCE MACHINERIES}

Comparison of the components of the replication machinery of retroviruses suggests some further relationships besides the RNase $\mathrm{H}$ with the PIWI domain. Several similarities are summarized in Table 1. The RT, for example, has an attachment site for the RNA primer for initiating cDNA synthesis by the primer grip (Jacques et al. 1994). A similar function has been described for the PAZ domain of AGO2, fixing the $3^{\prime}-\mathrm{OH}$ end of the antisense strand in a PAZ pocket. Furthermore, AGO proteins have a binding pocket for the phosphorylated $5^{\prime}$ end of the antisense strand (Ma et al. 2005) with no known equivalent for the RT/RNase $\mathrm{H}$.

The HIV RNase H requires RNA-binding properties similar to those of PIWI. Fusion of the RNase H to the RT domain supplies this ability. The RNase H without the RT domain has been shown to be active only in a special in situ assay by immobilizing the RNase $\mathrm{H}$ domain in a gel containing a radioactive hybrid (Schulze et al. 1991). Perhaps this assay may allow detection of RNase $\mathrm{H}$ activities of some of those AGO proteins, which appear to be inactive. Furthermore, the RT has an RNA-dependent as well as DNA-dependent DNA polymerase activity. It is not known whether it can also function as an RNAdependent RNA polymerase. The RT also has an unwind- 

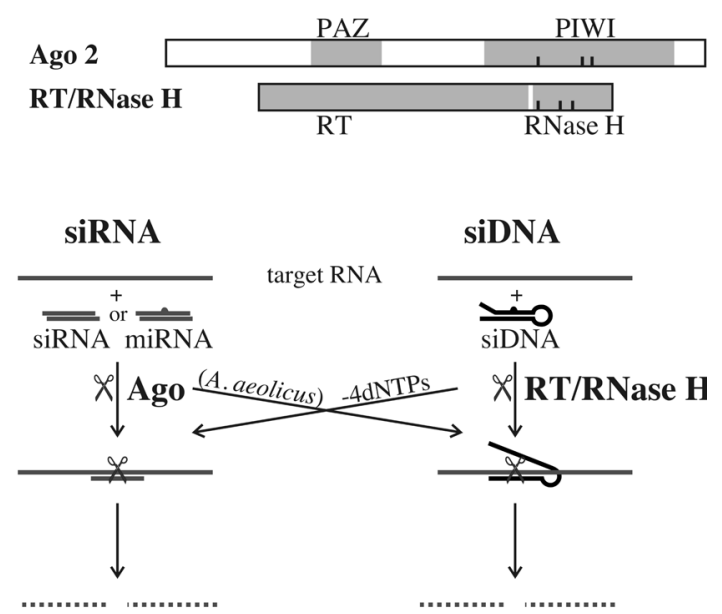

Antiviral defense

\section{No viral replication, suicide}

Figure 2. A relationship between siRNA components and retroviral proteins is proposed. PAZ and PIWI correspond to $\mathrm{RT} / \mathrm{RNase} \mathrm{H}$, and Dicer corresponds to integrase (see also Table 1). PIWI of AGO2 and RT/RNase $\mathrm{H}$ are functionally related. This is shown by siRNA-mediated RNA silencing in parallel to "siDNA" silencing of retroviral RNA. AGO2 and RT/RNase H can also cleave the other substrates with less specificity and lower efficiency. This has been shown with the archaebacterial AGO (Yuan et al. 2005) and RT/RNase H, whereby nonspecific cleavage was increased in the absence of 4dNTPs. HIV commits suicide with the "siDNA" targeted to the PPT, which prematurely activates the viral RT/RNase $\mathrm{H}$ for cleavage of the viral RNA before the first cDNA is made. Thus, the viral RNA is irreversibly destroyed (Moelling et al. 2006).

ing activity (Hottiger et al. 1994), corresponding to the helicase in the RNA interference machinery. Some similarities seem to exist between the integrase of retroviruses and Dicer, both of which generate dinucleotide 3'overhanging ends at the DNA, in the case of the DNA provirus at the two identical long terminal repeats (LTRs) and in the case of Dicer on dsRNA fragments. In addition, the integration site in the DNA of the host cell requires this type of cleavage. Both enzymes have nucleic-acidbinding domains. The integrase forms dimers for cleavage. Perhaps Dicer also dimerizes to produce 21-mers.

Table 1. Similarities of Retroviral Replication and RNA Interference Machineries

\begin{tabular}{ll}
\hline Retrovirus & siRNA \\
\hline Reverse transcriptase (RT) & PAZ \\
RNase H & PIWI \\
3'-OH, 5'-phosphate & 3'-OH, 5'-phosphate \\
oligonucleotide & oligonucleotide \\
RT (primer-grip) & PAZ pocket \\
RT (RNA-dependent DNA & RNA-dependent RNA \\
polymerase/DNA-dependent & polymerase \\
DNA polymerase) & \\
RT (DNA unwinding) & Helicase \\
Integrase (dinucleotides & Dicer (dinucleotides \\
overhangs) & overhangs) \\
Nucleocapsid (melting, & RNA-binding proteins, \\
RNA protection) & TRBP, FMRP \\
TRBP & TRBP \\
Protease & $?$ \\
\hline
\end{tabular}

Furthermore, retroviruses code for a nucleocapsid (NC) protein with a bipartite basic stretch of sequences, binding to single-stranded as well as double-stranded RNA. It is a chaperone or matchmaker and straightens out hairpinlooped structures and fixes the tRNA primer to the viral RNA at the primer-binding site (PBS). It may be worth noting that the NC has, in addition to its unspecific RNA-binding properties, the ability to specifically bind and select only one tRNA from the cellular pool, which requires a sequence-specific recognition through its zinc fingers (Dannull et al. 1994). RNA-binding domains or factors are also associated with the PAZ-PIWI domains of AGO2, even though a sequence specificity has not been detected. An RNA-binding protein is the fragile $\mathrm{X}$ syndrome mental retardation protein (FMRP), which can associate with AGO (Caudy et al. 2002). What about the protease, another highly specific and important component in the retroviral replication machinery? Is it yet to be found in siRNAmediated silencing? There is a protease involved in an antiviral defense mechanism, the virus fights back by a protein, which drives AGO into the proteasomal pathway in plants (D.C. Baulcombe, pers. comm.).

Structural components of retroviruses may not be related to the siRNA machinery, but what about some of the luxury functions known for HIV such as Tat, Rev, Nev, and Vif? The Tat RNA-binding protein (TRBP) is used by the virus (HIV) and the cell, where it can complex with AGO (Chendrimada et al. 2005).

\section{RNA VIRUSES ESCAPE THE HOST DEFENSE BY SUPPRESSORS OR BY A DNA PROVIRUS}

RNA viruses with their dsRNA intermediates are targets of the siRNA-mediated cellular defense, and hit back, for example, by P19 (Voinnet et al. 1999), p21 (Reed et al. 2003), or suppressor proteins counteracting AGO. Retroviruses may have escaped the siRNA-mediated antiviral defense and developed instead of suppressors a disguise as dsDNA proviruses via an RNA-DNA intermediate. They may have adopted some of the siRNA machinery for their replication machinery, specializing in tools for DNA instead of RNA. One cellular defense mechanism against an integrated DNA provirus can be by keeping it silent by DNA methylation, which in some species is mediated by small RNAs. The equivalent of this small RNA in mammals are methylases. The virus can escape this effect by fast replication.

If this also fails against viral invaders, the next level of defense is posttranscriptional gene silencing (PTGS), leading to destruction of the viral mRNA by small RNAs at noncoding untranscribed regions or inhibition of translation or initiation of translation. At this stage, DNA viruses may also be silenced. If the invader escaped even this level of defense, the ultimate rationale of the cell would be, while dying, to warn the neighboring cells by diffusion of small RNAs, thereby guaranteeing survival of an organism or a cell community, not the individual cell. Thus, diffusible small RNAs are the alarm system for bystander cells in plants. The worm Caenorhabditis elegans can even warn other worms in the neighborhood by secreted siRNAs. In mammals, such an effect is 
achieved probably not by siRNA but by interferon, a diffusible protein, which alarms bystander cells against viruses. There are other striking similarities between siRNA and the interferon response, the RNA-dependent protein kinase (PKR), which inhibits translation, and RNase L, which degrades mRNA. Thus, it appears that siRNA-mediated responses in primitive organisms have been replaced by proteins in the mammalian system.

\section{CONCLUSIONS}

The siRNA-mediated antiviral defense machinery in the RNA World may have coevolved with the retrovirus replication machinery. We and other investigators have shown a structure/function relationship between retroviral RT/RNase H and PIWI; we also pointed out the related properties of the two machineries. This analogy may raise questions or make predictions, e.g., whether the viral protease has an equivalent in the RNA interference system, yet to be discovered. Furthermore, we may not yet have determined the equivalent of the viral sequence-specific RNAbinding protein. Cellular defense against integrated DNA proviruses requires mechanisms such as siRNA-mediated methylation for silencing of the viral DNA in plants. The mammalian equivalent may occur via proteins such as methylases. Warning of neighboring cells or organisms by small RNAs may have evolved into the interferon system. Analogies to the mammalian interferon system, especially in this latter respect, are striking, whereby proteins became the mediators and may have replaced the small RNAs. In summary, some of the analogies discussed here may allow predictions for the RNA interference machinery from the retroviral replication machinery as well as from the interferon system, in particular on the role of proteins instead of siRNAs in the mammalian system.

\section{ACKNOWLEDGMENTS}

I am indebted to Dr. M. Lorger for help with some constructs and to Dr. J. Heinrich for support with the manuscript and figures.

\section{REFERENCES}

Ben-Artzi H., Zeelon E., Gorecki M., and Panet A. 1992. Doublestranded RNA-dependent RNase activity associated with human immunodeficiency virus type 1 reverse transcriptase. Proc. Natl. Acad. Sci. 89: 927.

Blain S.W. and Goff S.P. 1993. Nuclease activities of Moloney murine leukemia virus reverse transcriptase. Mutants with altered substrate specificities. J. Biol. Chem. 268: 23585.

Caudy A.A., Myers M., Hannon G.J., and Hammond S.M. 2002. Fragile X-related protein and VIG associate with the RNA interference machinery. Genes Dev. 16: 2491.

Chendrimada T.P., Gregory R.I., Kumaraswamy E., Norman J., Cooch N., Nishikura K., and Shiekhattar R. 2005. TRBP recruits the Dicer complex to Ago2 for microRNA processing and gene silencing. Nature 436: 740 .

Coffin J.M., Hughes S.H., and Varmus H.E., eds. 1997. Reverse transcriptase and the generation of retroviral DNA. In Retroviruses, chapter 4, p. 121. Cold Spring Harbor Laboratory Press, Cold Spring Harbor, New York.

Dannull J., Surovoy A., Jung G., and Moelling K. 1994. Specific binding of HIV-1 nucleocapsid protein to PSI RNA in vitro requires $\mathrm{N}$-terminal zinc finger and flanking basic amino acid residues. $E M B O ~ J . ~ 13: 1525$.

Götte M., Fackler S., Hermann T., Perola E., Cellai L., Gross H.J., Le Grice S.F.J., and Heumann H. 1995. HIV-1 reverse transcriptase-associated RNase H cleaves RNA/RNA in arrested complexes: Implications for the mechanism by which RNase $\mathrm{H}$ discriminates between RNA/RNA and RNA/DNA. EMBO J. 14: 833.

Hansen J., Schulze T., Mellert W., and Moelling K. 1988. Identification and characterization of HIV-specific RNase $\mathrm{H}$ by monoclonal antibody. EMBO J. 7: 239.

Hostomsky Z., Hughes S.H., Goff S.P., and Le Grice S.F. 1994. Redesignation of the RNase D activity associated with retroviral reverse transcriptase as RNase H. J. Virol. 68: 1970.

Hottiger M., Podust V.N., Thimmig R.L., McHenry C., and Hubscher U. 1994. Strand displacement activity of the human immunodeficiency virus type 1 reverse transcriptase heterodimer and its individual subunits. J. Biol. Chem. 269: 986.

Jacques P.S., Wohrl B.M., Ottmann M., Darlix J.L., and Le Grice S.F. 1994. Mutating the "primer grip" of p66 HIV-1 reverse transcriptase implicates tryptophan-229 in template-primer utilization. J. Biol. Chem. 269: 26472.

Jendis J., Strack B., and Moelling K. 1998. Inhibition of replication of drug-resistant HIV type 1 isolates by polypurine tractspecific oligodeoxynucleotide TFO A. AIDS Res. Hum. Retrovir. 14: 999.

Jendis J., Strack B., Volkmann S., Boni J., and Molling K. 1996. Inhibition of replication of fresh HIV type 1 patient isolates by a polypurine tract-specific self-complementary oligodeoxynucleotide. AIDS Res. Hum. Retrovir. 12: 1161.

Ma J.B., Yuan Y.R., Meister G., Pei Y., Tuschl T., and Patel D.J. 2005. Structural basis for 5 -end-specific recognition of guide RNA by the A. fulgidus Piwi protein. Nature 434: 666.

Matskevich A.A., Ziogas A., Heinrich J., Quast S., and Moelling K. 2006. Short partially double-stranded oligodeoxynucleotide induces reverse transcriptase-mediated cleavage of HIV RNA and abrogates infectivity of virions. AIDS Res. Hum. Retrovir. (in press).

Moelling K. 1975. Reverse transcriptase and RNase H: Present in a murine virus and in both subunts of an avian virus. Cold Spring Harbor Symp. Quant. Biol. 39: 969.

Moelling K., Abels S., Jendis J., Matskevich A., and Heinrich J. 2006. Silencing of HIV by hairpin-loop-structured DNA oligonucleotide. FEBS Lett. 580: 3545.

Moelling K., Bolognesi D.P., Bauer H., Büsen W., Plassmann H.W., and Hausen P. 1971. Association of the viral reverse transcriptase with an enzyme degrading the RNA moiety of RNA-DNA hybrids. Nat. New Biol. 234: 240.

Nowotny M., Gaidamakov S.A., Crouch R.J., and Yang W. 2005. Crystal structures of RNase H bound to an RNA/DNA hybrid: Substrate specificity and metal-dependent catalysis. Cell 121: 1005.

Ohtani N., Yanagawa H., Tomita M., and Itaya M. 2004. Cleavage of double-stranded RNA by RNase HI from a thermoacidophilic archaeon, Sulfolobus tokodaii 7. Nucleic Acids Res. 32: 5809 .

Reed J.C., Kasschau K.D., Prokhnevsky A.I., Gopinath K., Pogue G.P., Carrington J.C., and Dolja V.V. 2003. Suppressor of RNA silencing encoded by Beet yellows virus. Virology 306: 203.

Schulze T., Nawrath M., and Moelling K. 1991. Cleavage of the HIV-1 p66 reverse transcriptase/RNase $\mathrm{H}$ by the $\mathrm{p} 9$ protease in vitro generates active p15 RNase H. Arch. Virol. 118: 179.

Song J.J., Smith S.K., Hannon G.J., and Joshua-Tor L. 2004. Crystal structure of Argonaute and its implications for RISC slicer activity. Science 305: 1434.

Voinnet O., Pinto Y.M., and Baulcombe D.C. 1999. Suppression of gene silencing: A general strategy used by diverse DNA and RNA viruses of plants. Proc. Natl. Acad. Sci. 96: 14147.

Yang W. and Steitz T.A. 1995. Recombining the structures of HIV integrase, RuvC and RNase H. Structure 3: 131.

Yuan Y.R., Pei Y., Ma J.B., Kuryavyi V., Zhadina M., Meister G., Chen H.Y., Dauter Z., Tuschl T., and Patel D.J. 2005. Crystal structure of A. aeolicus argonaute, a site-specific DNA-guided endoribonuclease, provides insights into RISC-mediated mRNA cleavage. Mol. Cell 19: 405. 


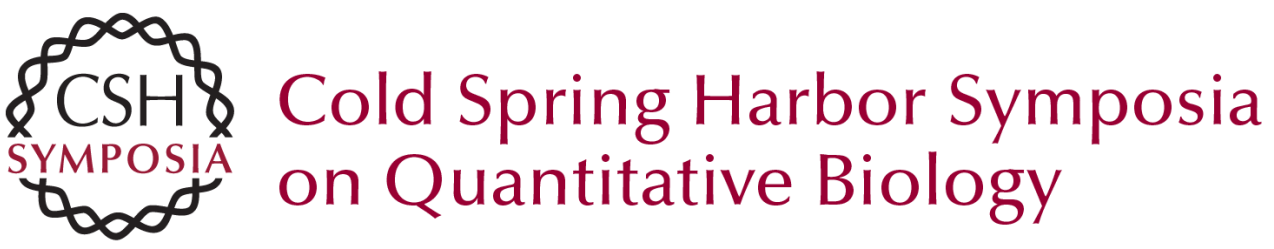

\section{Relationship between Retroviral Replication and RNA Interference Machineries}

K. MOELLING, A. MATSKEVICH and J.-S. JUNG

Cold Spring Harb Symp Quant Biol 2006 71: 365-368

Access the most recent version at doi:10.1101/sqb.2006.71.010

References This article cites 24 articles, 7 of which can be accessed free at:

http://symposium.cshlp.org/content/71/365.full.html\#ref-list-1

License

Email Alerting Receive free email alerts when new articles cite this article - sign up in

Service the box at the top right corner of the article or click here. 